\title{
Self-rotation and synchronization in exciton-polariton condensates
}

\author{
Hiroki Saito ${ }^{1}$ and Rina Kanamoto ${ }^{2}$ \\ ${ }^{1}$ Department of Engineering Science, University of Electro-Communications, Tokyo 182-8585, Japan \\ ${ }^{2}$ Department of Physics, Meiji University, Kawasaki, Kanagawa 214-8571, Japan
}

(Dated: October 1, 2018)

\begin{abstract}
Self-rotation occurs in an exciton-polariton condensate in a two-dimensional semiconductor microcavity pumped by a nonresonant Gaussian laser beam. A wave packet of the condensate spontaneously rotates around the center of the pumped region at a constant frequency breaking the rotation symmetry of the system. When two self-rotating condensates are created with an appropriate distance, synchronization occurs between the dynamics of the self-rotating condensates.
\end{abstract}

\section{INTRODUCTION}

Self-oscillations are ubiquitous phenomena that occur in nature, ranging from whistles and heartbeats to pulsating variable stars 1 . Self-oscillation is induced by some instability and maintained by the oscillation itself, i.e., external energy is synchronously taken into the system, which balances with dissipation, and results in the stable limit cycle in the phase space. When two or more selfoscillators are coupled with each other, synchronization can occur between their oscillations, as Huygens observed in pendulum clocks ${ }^{2}$.

Self-oscillation can be observed in open quantum systems with nonlinearity, in which energy is replenished from and dissipated into their environments. Laser and Josephson oscillations may be regarded as selfoscillations in quantum systems. Self-oscillations have also been observed in optomechanical systems 3,4 and in a resonator driven by a superconducting single-electron transistor $^{5}$. Here, we focus on exciton-polariton condensates in a planar microcavity ${ }^{6.7}$ as an open quantum system to investigate the self-oscillation. Excitonpolaritons excited by external laser beams have a lifetime of picoseconds, which is of the same order as the characteristic time scale of polariton dynamics. Therefore, the exciton-polariton condensate is a nonequilibrium open system. Various experiments have been performed on this system, such as observations of BoseEinstein condensation ${ }^{8}$, quantized vortices ${ }^{9,10}$, quantum hydrodynamics ${ }^{11-14}$, and a chaotic non-Hermitian billiard ${ }^{15}$. Self-oscillations and synchronization in excitonpolariton condensates have been predicted in Refs. 16 23, and the phase locking of condensates has been reported in Ref. 24, 25. The Bénard-von Kármán vortex street in an exciton-polariton superfluid ${ }^{26}$ can also be regarded as self-oscillation.

In this paper, we show that a wave packet of an exciton-polariton condensate that is nonresonantly pumped by a Gaussian laser beam rotates spontaneously around the center of the pumped region. This selfrotation is a consequence of spontaneous breaking of the rotation and clockwise-counterclockwise symmetries, and the system acquires an angular momentum. We also show that when two self-rotating condensates are excited by two Gaussian laser beams with an appropriate distance between them, the rotating dynamics are synchronized, even when the individual rotation frequency is different.

This paper is organized as follows. The problem is formulated and the properties of the uniform system are reviewed in Sec. III. The self-rotation of the condensate is investigated in Sec. III. The synchronization of two self-rotating condensates is demonstrated in Sec. IV] A conclusion and discussion are provided in Sec. V.

\section{MODULATIONAL INSTABILITY IN A HOMOGENEOUS SYSTEM}

First, we formulate the problem and give a brief review of the modulational instability in a homogeneous system. The dynamics of an exciton-polariton condensate with an effective mass $m$ is modeled by the nonlinear Schrödinger equation given by 27

$i \hbar \frac{\partial \psi}{\partial t}=-\frac{\hbar^{2}}{2 m} \nabla^{2} \psi+\frac{i \hbar}{2}\left(R n_{R}-\gamma_{c}\right) \psi+g_{c}|\psi|^{2} \psi+g_{R} n_{R} \psi$

where $\psi(\boldsymbol{r}, t)$ is the macroscopic wave function of the exciton-polariton condensate and $n_{R}(\boldsymbol{r}, t)$ represents the polariton density of the reservoir, which obeys

$$
\frac{\partial n_{R}}{\partial t}=P-\gamma_{R} n_{R}-R n_{R}|\psi|^{2} .
$$

The external laser pump $P$ creates polaritons with much larger energy than that of the condensate polaritons in the lower branch. The pumped polaritons then relax and form the reservoir $n_{R}$, which feeds polaritons to the condensate with a rate $R$. In this section, we consider a homogeneous pump with a constant $P$. The loss rates of the condensate and reservoir polaritons are $\gamma_{c}$ and $\gamma_{R}$, respectively. In Eq. (11), the condensate polaritons interact with each other through the nonlinear term with a coefficient $g_{c}$, and the interaction between the condensate and reservoir polaritons is described by the term with $g_{R}$.

In a homogeneous system, the polariton densities in the steady state above the condensation threshold are given by $\left|\psi_{0}\right|^{2}=\left(P-P_{\mathrm{th}}\right) / \gamma_{c}$ and $n_{R 0}=\gamma_{c} / R$, where $P_{\mathrm{th}}=$ $\gamma_{c} \gamma_{R} / R$. This homogeneous solution is modulationally unstable for a weak pumping regime ${ }^{20,27-29}$, which can 


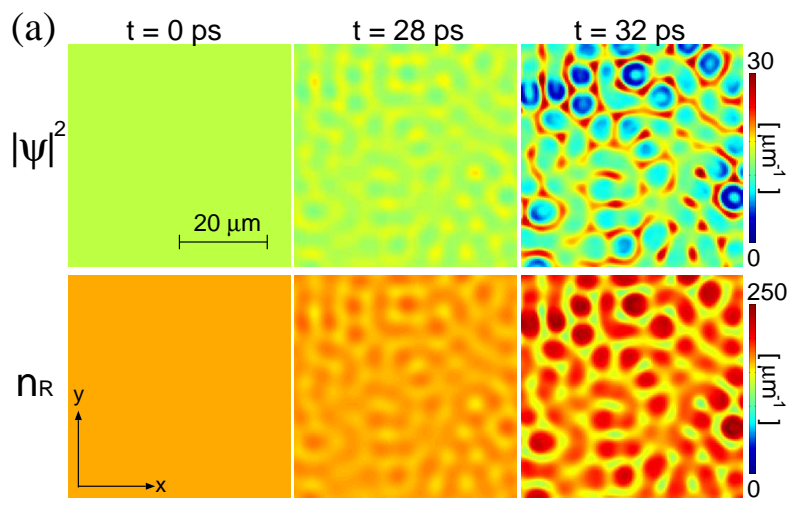

(b)

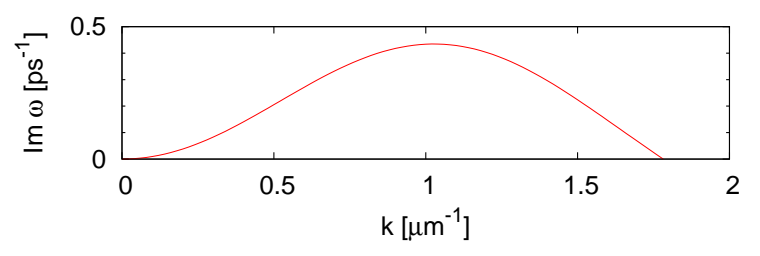

FIG. 1: (color online) Modulational instability in the uniform system with $\gamma_{c}^{-1}=0.15 \mathrm{ps}, \gamma_{R}^{-1}=3 \mathrm{ps}, g_{c}=6 \times 10^{-3} \mathrm{meV}$. $\mu \mathrm{m}^{2}, g_{R}=2 g_{c}, R=0.04 \mathrm{ps}^{-1} \mu \mathrm{m}^{2}, P=160 \mathrm{ps}^{-1} \mu \mathrm{m}^{-2} \simeq$ $2.9 P_{\mathrm{th}}$, and $m=5 \times 10^{-5}$ electron mass. (a) Time evolution of the condensate and reservoir density profiles, $|\psi|^{2}$ and $n_{R}$, where the initial state is the stationary state. (b) Positive imaginary part of the Bogoliubov spectrum.

be explained by the effective attractive interaction in the condensate $\frac{28}{2}$. Figure 1(a) shows the dynamics of the density profiles under modulational instability, where the initial state is $\psi=\psi_{0}$ and $n_{R}=n_{R 0}$ with a small random noise, which is a random number set on each numerical mesh. In this and the following numerical simulations, the pseudospectral method with the fourth-order RungeKutta scheme is employed. The unstable wavelengths can be obtained from the Bogoliubov analysis. Substituting $\psi(\boldsymbol{r}, t)=\left[\psi_{0}+u_{k} e^{i \boldsymbol{k} \cdot \boldsymbol{r}-i \omega t}+v_{k}^{*} e^{-i \boldsymbol{k} \cdot \boldsymbol{r}-i \omega^{*} t}\right] e^{-i \mu t / \hbar}$ and $n_{R}(\boldsymbol{r}, t)=n_{R 0}+\operatorname{Re}\left(n_{k} e^{i \boldsymbol{k} \cdot \boldsymbol{r}-i \omega t}\right)$ with $\mu=g_{c}\left|\psi_{0}\right|^{2}+$ $g_{R} n_{R 0}$ into Eqs. (1) and (2) and diagonalizing the $3 \times$ 3 matrix, we obtain the Bogoliubov spectrum $\omega(k)$ and mode coefficients $u_{k}, v_{k}$, and $n_{k}$. When the imaginary part of $\omega(k)$ is positive, the mode with the wave number $k$ is unstable. Figure 1(b) shows the imaginary part of $\omega(k)$. The most unstable wavelength is $\lambda \simeq 2 \pi \mu \mathrm{m}$, which agrees with the pattern in Fig. 11(a).

\section{SPONTANEOUS ROTATION WITH A GAUSSIAN PUMP}

We consider a situation in which exciton-polaritons are pumped by a Gaussian laser beam. The pump profile is given by

$$
P(\boldsymbol{r})=P_{0} e^{-r^{2} / \rho^{2}},
$$
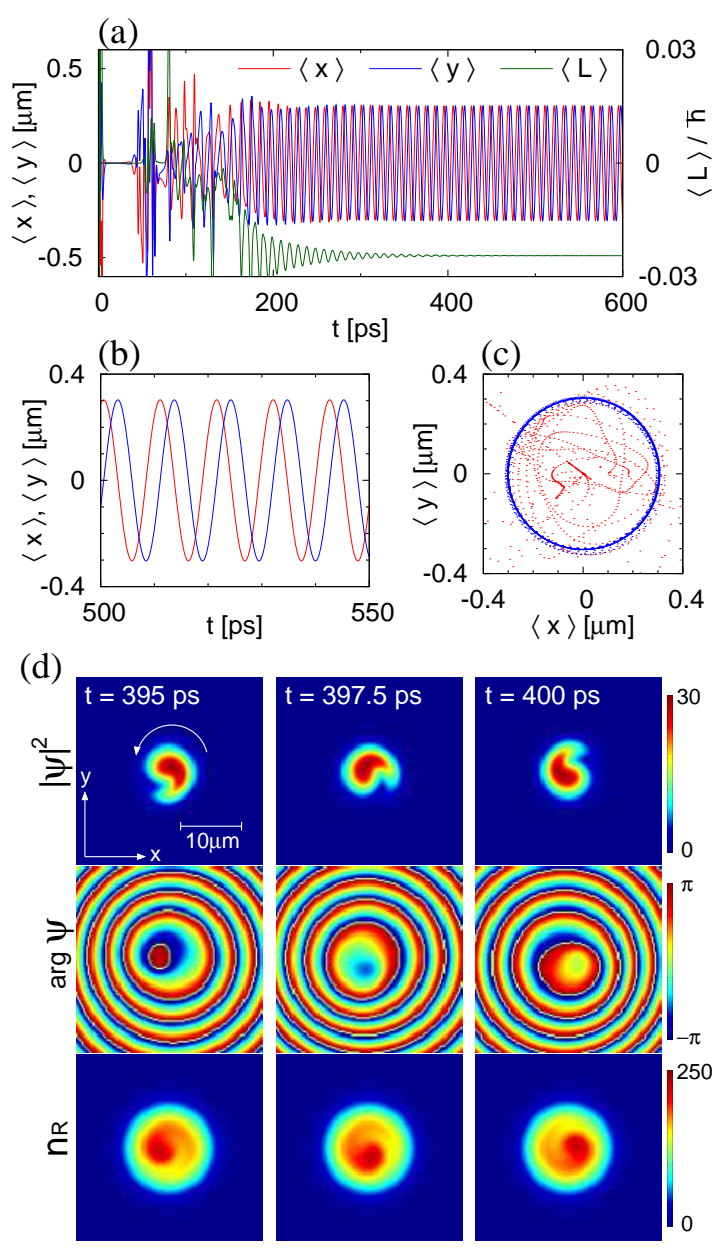

FIG. 2: (color online) Time evolution of the system pumped by a Gaussian laser beam with $P_{0}=160 \mathrm{ps}^{-1} \mu \mathrm{m}^{-2}$ and $\rho=5$ $\mu \mathrm{m}$. The other parameters are the same as those used in Fig. 1 (a) Time evolution of the center-of-mass (COM) position $(\langle x\rangle,\langle y\rangle)$ and the angular momentum $\langle L\rangle$ of the condensate. (b) Magnification of (a). (c) Trajectory of $(\langle x\rangle,\langle y\rangle)$, where the red and blue points are $t<200 \mathrm{ps}$ and $t>200 \mathrm{ps}$, respectively. The points are plotted every $0.17 \mathrm{ps}$. (d) Snapshots of the density and phase profiles, $|\psi|^{2}, \arg \psi$, and $n_{R}$. The white arrow indicates the direction of rotation for the COM position. See the Supplemental Material for a movie of the condensate dynamics 30 .

where $P_{0}$ is the peak intensity and $\rho$ is the $1 / e$ width of the beam. When the pump width $\rho$ is much larger than the unstable wavelengths of a homogeneous system pumped by $P=P_{0}$, the central region of the pump is sufficiently wide for modulational instability and pattern formation. On the other hand, when $\rho$ is much smaller than the unstable wavelengths, no modulational instability occurs in the Gaussian-shaped condensate. An interesting situation is expected for $\rho$ comparable to the unstable wavelength. Self-oscillations have been observed in a one-dimensional system in this regime ${ }^{20}$.

Figure 2 shows the dynamics of the system pumped by a Gaussian laser beam with $\rho=5 \mu \mathrm{m}$, where the 
initial state is $\psi=n_{R}=0$ plus a small random noise. Figures 2(a)-2(c) show the time evolution of the centerof-mass (COM) position,

$$
\langle\boldsymbol{r}\rangle=\frac{\int \boldsymbol{r}|\psi|^{2} d \boldsymbol{r}}{\int|\psi|^{2} d \boldsymbol{r}}
$$

and the angular momentum per particle,

$$
\langle L\rangle=\frac{-i \hbar \int \psi^{*}\left(x \partial_{y}-y \partial_{x}\right) \psi d \boldsymbol{r}}{\int|\psi|^{2} d \boldsymbol{r}}
$$

of the condensate. After $t \sim 200 \mathrm{ps}$, the COM motion becomes periodic and the angular momentum converges to a constant value. The trajectory of $\langle\boldsymbol{r}\rangle$ is entrained into a circle and rotates along a circle at a constant angular frequency. This circular trajectory is robust against external perturbation. We have confirmed that the trajectory always returns to the circle after the regular dynamics are disturbed by a temporal external potential.

Figure 2(d) shows the time evolution of the density and phase profiles for the regular motion. A tadpole-shaped condensate wave packet rotates around the center counterclockwise. It is interesting to note that the angular momentum $\langle L\rangle$ is negative, whereas the tadpole-shaped wave packet rotates counterclockwise. This seeming contradiction arises from the superflow in the condensate. Figure 2(d) shows $\arg \psi$, which indicates that the superfluid flows out from the point near the head of the tadpole, and results in superflow from the head to the tail of the tadpole. This is because the condensate is fed from the reservoir mainly at the head of the tadpole, because the density of the reservoir is large ahead of the tadpole. As a result, the tadpole grows ahead, while the tail is always shrunk by the dissipation. This is the reason why the directions of the tadpole rotation and the angular momentum are opposite. For initial noises with different random seeds, $\langle L\rangle>0$ and $\langle L\rangle<0$ occurs with the same probability due to the chiral symmetry of the system.

The pump with a Gaussian beam thus induces the rotation dynamics, which can be regarded as self-rotation, because the rotation symmetry of the system is spontaneously broken and the stable rotation persists without any external rotating force. The rotation is driven by the interplay between the condensate and reservoir; the tadpole-shaped condensate grows ahead due to the density gradient in the reservoir, while the high density region of the reservoir chases the tail of the tadpole where the supply to the condensate is small.

Figure 3 shows the parameter dependence of the period of the self-rotation. The self-rotation frequency increases with $P_{0}, R$, and $g_{R}$, and decreases as $\gamma_{c}, \gamma_{R}$, and $g_{c}$ increase.

\section{SYNCHRONIZATION BETWEEN TWO ROTATING CONDENSATES}

We study the case in which two self-rotating condensates are excited by two Gaussian laser beams, and ex-
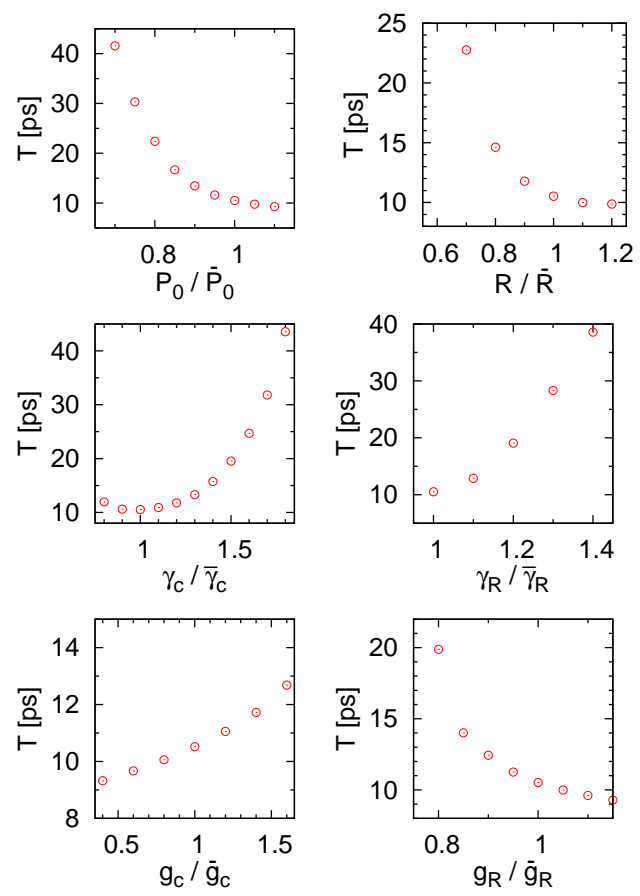

FIG. 3: (color online) Parameter dependence of the period $T$ of the self-rotation. The parameters used in Fig. 2 are denoted as $\bar{P}_{0}, \bar{R}, \bar{\gamma}_{c}, \bar{\gamma}_{R}, \bar{g}_{c}$, and $\bar{g}_{R}$.

amine whether synchronization occurs between the two self-rotations. The external pump is given by

$$
P(\boldsymbol{r})=P_{\text {left }} e^{-\left(\boldsymbol{r}-\boldsymbol{R}_{\text {left }}\right)^{2} / \rho^{2}}+P_{\text {right }} e^{-\left(\boldsymbol{r}-\boldsymbol{R}_{\text {right }}\right)^{2} / \rho^{2}},
$$

where $P_{\text {left,right }}$ and $\boldsymbol{R}_{\text {left,right }}=\left(X_{\text {left,right }}, Y_{\text {left,right }}\right)$ are the peak intensities and the positions of the two Gaussian-shaped pumps, respectively. Without loss of generality, we set $Y_{\text {left,right }}=0$. The pump intensities are taken to be $P_{\text {left }}=160 \mathrm{ps}^{-1} \mu \mathrm{m}^{-2}$ and $P_{\text {right }}=0.99 P_{\text {left }}$, which results in different self-rotation frequencies (see Fig. 3). If the two self-rotating condensates are independent (i.e. in the case of $X_{\text {right }}-X_{\text {left }} \rightarrow \infty$ ), then the rotation period is $10.52 \mathrm{ps}$ for $P_{\text {left }}$ and $10.70 \mathrm{ps}$ for $P_{\text {right }}$. The COM position of each condensate is defined relative to the center of the pump as

$$
\langle\boldsymbol{r}\rangle_{\text {left,right }}=\frac{\int_{\left|\boldsymbol{r}-\boldsymbol{R}_{\text {left,right }}\right|<r_{c}}\left(\boldsymbol{r}-\boldsymbol{R}_{\text {left,right }}\right)|\psi|^{2} d \boldsymbol{r}}{\int_{\left|\boldsymbol{r}-\boldsymbol{R}_{\text {left,right }}\right|<r_{c}}|\psi|^{2} d \boldsymbol{r}},
$$

where the cutoff radius $r_{c}$ is taken to be $8 \mu \mathrm{m}$, which is sufficient to cover each condensate.

Figure 4(a) shows the time evolution of the two selfrotating condensates, where the distance between the two pumps is $X_{\text {right }}-X_{\text {left }}=20 \mu \mathrm{m}$. In this case, the two condensates are well separated from each other and the interaction between them is negligible. In Fig. 4(a), the two self-rotations are in opposite directions, while they also rotate in the same direction depending on the initial noise. The left- and right-side condensates rotate 

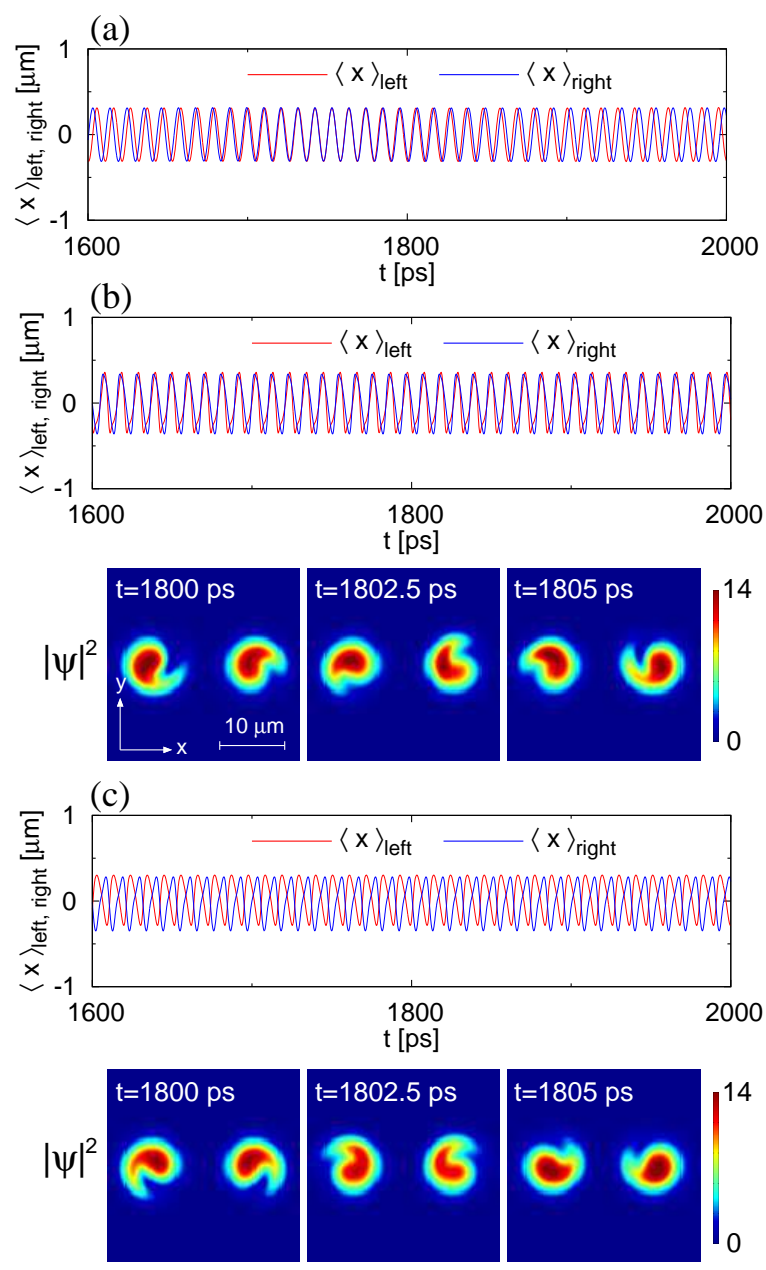

FIG. 4: (color online) Dynamics of two self-rotating condensates pumped according to Eq. (6) with $P_{\text {left }}=160 \mathrm{ps}^{-1} \mu \mathrm{m}^{-2}$ and $P_{\text {right }}=0.99 P_{\text {left }}$. The other parameters are the same as those in Fig. 2. (a) Time evolution of $\langle x\rangle_{\text {left }}$ and $\langle x\rangle_{\text {right }}$ with $X_{\text {right }}-X_{\text {left }}=20 \mu \mathrm{m}$. The two self-rotations are not synchronized. (b) Time evolution of $\langle x\rangle_{\text {left }}$ and $\langle x\rangle_{\text {right }}$ (upper panel) and snapshots of the density profile $|\psi|^{2}$ (lower panels), where $X_{\text {right }}-X_{\text {left }}=16 \mu \mathrm{m}$. Synchronization occurs between the two self-rotating condensates. (c) The same conditions as those in (b), except a random number seed used to produce the initial noise. See the Supplemental Material for movies of the dynamics in (b) and (c) ${ }^{30}$.

independently with periods of 10.52 and $10.70 \mathrm{ps}$, respectively, and no synchronization occurs. Figure 4(b) shows the synchronization for $X_{\text {right }}-X_{\text {left }}=16 \mu \mathrm{m}$. The COM positions $\langle x\rangle_{\text {left }}$ and $\langle x\rangle_{\text {right }}$ oscillate in phase at the same frequency. The two condensates rotate in opposite directions with a common rotation period of $10.53 \mathrm{ps}$, by which it seems that the right-side frequency is entrained into the left-side frequency $\stackrel{31}{ }$. Interestingly, another type of synchronization is observed for the same parameters, which is shown in Fig. 4 (c). The COM positions $\langle x\rangle_{\text {left }}$ and $\langle x\rangle_{\text {right }}$ oscillate out of phase with a period of 10.55 ps. The only difference between the numerical simula-
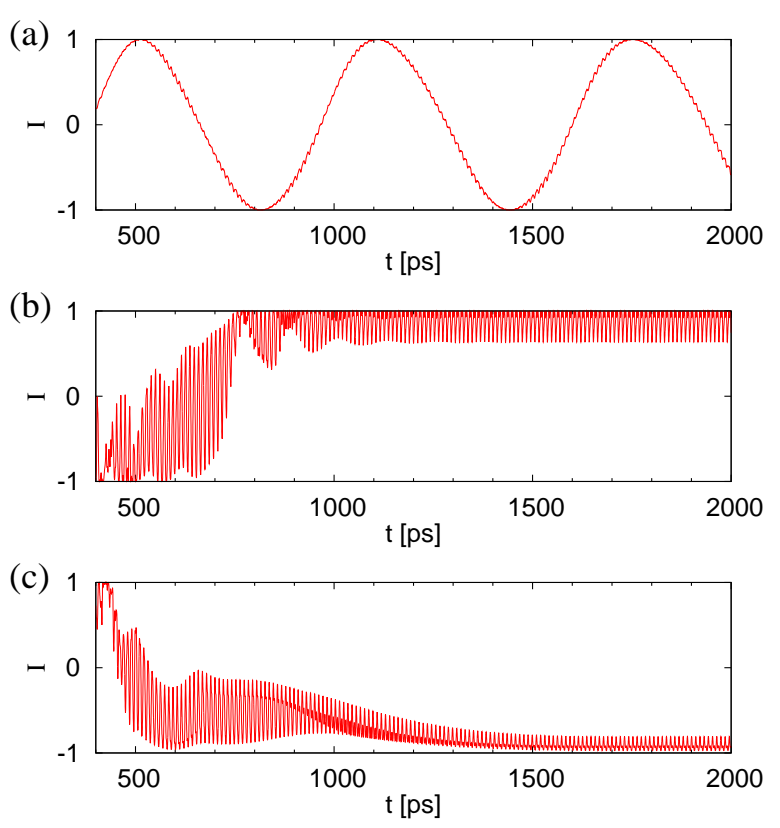

FIG. 5: (color online) Time evolution of $I$ defined in Eq. (8), where (a)-(c) correspond to the dynamics shown in Figs. 4(a)4(c).

tions in Fig. 4(b) and 4(c) is the random number seed used to produce the small initial noise.

To clearly visualize the synchronization, the inner product is calculated:

$$
I=\hat{x}_{\text {left }} \hat{x}_{\text {right }}-\hat{y}_{\text {left }} \hat{y}_{\text {right }},
$$

where $\hat{\boldsymbol{r}}_{\text {left,right }}=\langle\boldsymbol{r}\rangle_{\text {left,right }} /\left|\langle\boldsymbol{r}\rangle_{\text {left,right }}\right|$ are the unit vectors of the COM positions relative to the centers of the pumps. The minus sign in Eq. (8) is introduced to quantify the synchronization between the opposite rotations, as shown in Figs. 4(b) and 4(c). If the two unit vectors rotate in the opposite directions as $x_{\text {left }}+i y_{\text {left }}=e^{i \omega t}$ and $x_{\text {right }}+i y_{\text {right }}=e^{-i \omega^{\prime} t+\phi}$, then $I=\cos \left[\left(\omega-\omega^{\prime}\right) t+\phi\right]$. When frequency lock occurs, $I$ becomes constant.

Figures $5(\mathrm{a})-5(\mathrm{~s})$ show the time evolution of $I$, which correspond to the dynamics shown in Figs. 4(a)-4(c). When the two self-rotations are not synchronized, $I$ oscillates at their frequency difference, as shown in Fig. 5(a), where the period is $\simeq\left(10.52^{-1}-10.7^{-1}\right)^{-1} \simeq 625$ ps. When synchronization occurs, $I$ oscillates around a constant value, as shown in Figs. 5(b) and 5(c). The synchronized phase $\phi$ is $\simeq 0$ in Fig. 5 (b) and $\simeq \pi$ in Fig. 5 (c).

\section{CONCLUSIONS AND DISCUSSION}

We have investigated the dynamics of an excitonpolariton condensate in a planar semiconductor microcavity pumped by a nonresonant laser beam. We considered a parameter region in which modulational instability arises in a homogeneous system (Fig. 1). It was 
shown that when the polariton condensate is excited by a Gaussian laser beam of which the width is comparable to the unstable wavelength, the self-rotation of the condensate occurs; the COM position of the condensate wave packet rotates around the center of the pump at a constant frequency (Fig. 2). The parameter dependence of the self-rotation frequency was investigated (Fig. 33). In addition, the synchronization of two self-rotating condensates pumped by two Gaussian laser beams was investigated (Figs. 4 and [5). The two self-rotations could be synchronized, even when the individual rotation frequencies were slightly different.

Experimentally, there always exist imperfections in a sample of the semiconductor microcavity, which produces random potential for polaritons. We have performed numerical simulations including random potential with a typical energy of $\sim 0.1 \mathrm{meV}$ and spatial scale of $\sim 10$ $\mu \mathrm{m}$, and confirmed that the self-rotation and the synchronization occur even in the presence of the disorder potential.

Although it was assumed that $\gamma_{c} \gg \gamma_{R}$ in this work, the loss rates satisfy $\gamma_{c} \lesssim \gamma_{R}$ for typical samples in the experiments. The condition $\gamma_{c} \gg \gamma_{R}$ may be satisfied by a reduction of the Q-factor of the microcavity at the frequency of the condensate polariton, which may be realized near the edge of the stopband of the distributed Bragg reflector.

Spontaneous rotation has been studied for the nonlinear Schrödinger equation with pump and decay ${ }^{32}$, and recently a wave packet of a condensate in a harmonic potential was found to rotate spontaneously ${ }^{33}$. The mechanism for these spontaneous rotations may be different from that presented here, because only the condensate wave function was considered in these previous studies, while the interplay between the condensate and the reservoir plays an important role in the self-rotation in this work.

\section{Acknowledgments}

The authors thank S. Kaneko for his participation in the early stage of this work. This work was supported by JSPS KAKENHI Grant Numbers JP16K05505, JP26400414, and JP25103007.
1 A. Jenkins, Phys. Rep. 525, 167 (2013).

2 C. Huygens, "Oeuvres complétes de Christiaan Huygens, vol. 5" ed. D. B. de Haan (Martinus Nijhoff, Den Haag, 1893).

3 T. Carmon, H. Rokhsari, L. Yang, T. J. Kippenberg, and K. J. Vahala, Phys. Rev. Lett. 94, 223902 (2005).

4 T. J. Kippenberg, H. Rokhsari, T. Carmon, A. Scherer, and K. J. Vahala, Phys. Rev. Lett. 95, 033901 (2005).

5 D. A. Rodrigues, J. Imbers, and A. D. Armour, Phys. Rev. Lett. 98, 067204 (2007).

6 I. Carusotto and C. Ciuti, Rev. Mod. Phys. 85, 299 (2013).

7 T. Byrnes, N. Y. Kim, and Y. Yamamoto, Nat. Phys. 10, 803 (2014).

8 J. Kasprzak, M. Richard, S. Kundermann, A. Baas, P. Jeambrun, J. M. J. Keeling, F. M. Marchetti, M. H. Szymańska, R. André, J. L. Staehli, V. Savona, P. B. Littlewood, B. Deveaud, and Le Si Dang, Nature (London) 443, 409 (2006).

${ }^{9}$ K. G. Lagoudakis, M. Wouters, M. Richard, A. Baas, I. Carusotto, R. André, Le Si Dang, and B. Deveaut-Plédran, Nat. Phys. 4, 706 (2008).

10 G. Roumpos, M. D. Fraser, A. Löffler, S. Höfling, A. Forchel, and Y. Yamamoto, Nat. Phys. 7, 129 (2011).

11 A. Amo, D. Sanvitto, F. P. Laussy, D. Ballarini, E. del Valle, M. D. Martin, A. Lemaître, J. Bloch, D. N. Krizhanovskii, M. S. Skolnick, C. Tejedor, and L. Viña, Nature (London) 457, 291 (2009).

12 G. Nardin, G. Grosso, Y. Léger, B. Pietka, F. MorierGenoud, and B. Deveaut-Plédran, Nat. Phys. 7, 635 (2011).

13 A. Amo, S. Pigeon, D. Sanvitto, V. G. Sala, R. Hivet, I. Carusotto, F. Pisanello, G. Leménager, R. Houdré, E Giacobino, C. Ciuti, and A. Bramati, Science 332, 1167 (2011).
14 G. Grosso, G. Nardin, F. Morier-Genoud, Y. Léger, and B. Deveaud-Plédran, Phys. Rev. Lett. 107, 245301 (2011).

15 T. Gao, E. Estrecho, K. Y. Bliokh, T. C. H. Liew, M. D. Fraser, S. Brodbeck, M. Kamp, C. Schneider, S. Höfling, Y. Yamamoto, F. Nori, Y. S. Kivshar, A. G. Truscott, R. G. Dall, and E. A. Ostrovskaya, Nature (London) 526, 554 (2015).

16 M. Wouters, Phys. Rev. B 77, 121302(R) (2008).

17 P. R. Eastham, Phys. Rev. B 78, 035319 (2008).

18 M. O. Borgh, J. Keeling, and N. G. Berloff, Phys. Rev. B 81, 235302 (2010).

19 D. Read, Y. G. Rubo, and A. V. Kavokin, Phys. Rev. B 81, 235315 (2010).

20 N. Bobrovska, E. A. Ostrovskaya, and M. Matuszewski, Phys. Rev. B 90, 205304 (2014).

21 K. Rayanov, B. L. Altshuler, Y. G. Rubo, and S. Flach, Phys. Rev. Lett. 114, 193901 (2015).

${ }^{22}$ X. Ma, I. Yu. Chestnov, M. V. Charukhchyan, A. P. Alodjants, and O. A. Egorov, Phys. Rev. B 91, 214301 (2015).

23 T.-W. Chen, S.-D. Jheng, W.-F. Hsieh, S.-C. Cheng, Superlattices and Microstructures 92, 190 (2016).

24 A. Baas, K. G. Lagoudakis, M. Richard, R. André, Le Si Dang, and B. Deveaud-Plédran, Phys. Rev. Lett. 100, 170401 (2008).

${ }^{25}$ K. G. Lagoudakis, B. Pietka, M. Wouters, R. André, and B. Deveaud-Plédran, Phys. Rev. Lett. 105, 120403 (2010).

${ }^{26}$ H. Saito, T. Aioi, and T. Kadokura, Phys. Rev. B 86, 014504 (2012).

27 M. Wouters and I. Carusotto, Phys. Rev. Lett. 99, 140402 (2007).

28 L. A. Smirnov, D. A. Smirnova, E. A. Ostrovskaya, and Y. S. Kivshar, Phys. Rev. B 89, 235310 (2014).

29 T. C. H. Liew, O. A. Egorov, M. Matuszewski, O. Kyriienko, X. Ma, and E. A. Ostrovskaya, Phys. Rev. B 91, 
$085413(2015)$

30 See Supplemental Material at http:... for movies of the condensate dynamics.

31 A. Pikovsky, M. Rosenblum, and J. Kurths, "Synchronization: a universal concept in nonlinear sciences" (Cambridge University Press, Cambridge, 2001).
32 J. Keeling and N. G. Berloff, Phys. Rev. Lett. 100, 250401 (2008).

33 O. L. Berman, R. Ya. Kezerashvili, G. V. Kolmakov, and L. M. Pomirchi, Phys. Rev. E 91, 062901 (2015). 